quatrième série-tome 46 fascicule 3 mai-juin 2013

$$
\begin{aligned}
& \text { CANNALES } \\
& \text { SCIENTIFIQUES } \\
& \text { de } \\
& \text { L'ÉCOLE } \\
& \text { NORMALE } \\
& \text { SUPÉRIEURE }
\end{aligned}
$$

Anders S. BUCH \& Pierre-Emmanuel CHAPUT \& Leonardo C. MIHALCEA \& Nicolas PERRIN Finiteness of cominuscule quantum $K$-theory 
Ann. Scient. Éc. Norm. Sup.

$4^{\text {e }}$ série, t. 46,2013 , p. 477 à 494

\title{
FINITENESS OF COMINUSCULE QUANTUM $K$-THEORY
}

\author{
By Anders S. BUCH, Pierre-Emmanuel CHAPUT, \\ Leonardo C. MIHAlCEA and Nicolas PERRIN
}

\begin{abstract}
The product of two Schubert classes in the quantum $K$-theory ring of a homogeneous space $X=G / P$ is a formal power series with coefficients in the Grothendieck ring of algebraic vector bundles on $X$. We show that if $X$ is cominuscule, then this power series has only finitely many non-zero terms. The proof is based on a geometric study of boundary Gromov-Witten varieties in the Kontsevich moduli space, consisting of stable maps to $X$ that take the marked points to general Schubert varieties and whose domains are reducible curves of genus zero. We show that all such varieties have rational singularities, and that boundary Gromov-Witten varieties defined by two Schubert varieties are either empty or unirational. We also prove a relative Kleiman-Bertini theorem for rational singularities, which is of independent interest. A key result is that when $X$ is cominuscule, all boundary Gromov-Witten varieties defined by three single points in $X$ are rationally connected.
\end{abstract}

RÉsumé. - Le produit de deux classes de Schubert dans l'anneau de $K$-théorie quantique d'un espace homogène $X=G / P$ est une série formelle à coefficients dans l'anneau de Grothendieck des fibrés vectoriels algébriques au-dessus de $X$. Nous montrons que pour $X$ cominuscule, cette série formelle n'a qu'un nombre fini de termes non nuls. La preuve repose sur une étude géométrique de certaines variétés de Gromov-Witten contenues dans le bord de l'espace de modules de Kontsevitch. Ces variétés paramètrent des applications stables à valeurs dans $X$, dont la courbe source est une union réductible de courbes rationnelles, et qui envoient les points marqués dans des sous-variétés de Schubert générales. Nous montrons que ces variétés de Gromov-Witten sont à singularités rationnelles et que celles définies par seulement deux sous-variétés de Schubert sont soit vides soit unirationnelles. Nous présentons également un énoncé relatif, de type Kleiman-Bertini pour les singularités rationnelles, d'intérêt indépendant. Un résultat-clé pour notre preuve est le fait que toutes les variétés de Gromov-Witten du bord de l'espace de modules de Kontsevitch, définies par trois variétés de Schubert ponctuelles dans $X$, sont rationnellement connexes.

The first author was supported in part by NSF Grant DMS-0906148. 


\section{Introduction}

The goal of this paper is to prove that any product of Schubert classes in the quantum $K$-theory ring of a cominuscule homogeneous space contains only finitely many non-zero terms.

Let $X=G / P$ be a homogeneous space defined by a semisimple complex Lie group $G$ and a parabolic subgroup $P$, and let $\bar{M}_{0, n}(X, d)$ denote the Kontsevich moduli space of $n$-pointed stable maps to $X$ of degree $d$, with total evaluation map ev $: \bar{M}_{0, n}(X, d) \rightarrow X^{n}$. Given Schubert varieties $\Omega_{1}, \ldots, \Omega_{n} \subset X$ in general position, there is a Gromov-Witten variety $\mathrm{ev}^{-1}\left(\Omega_{1} \times \cdots \times \Omega_{n}\right) \subset \overline{\mathcal{M}}_{0, n}(X, d)$, consisting of all stable maps that send the $i$-th marked point into $\Omega_{i}$ for each $i$. The Kontsevich space and its Gromov-Witten varieties are the foundation of the quantum cohomology ring of $X$, whose structure constants are the (cohomological) Gromov-Witten invariants, defined as the number of points in finite Gromov-Witten varieties. More generally, the $K$-theoretic Gromov-Witten invariant $I_{d}\left(\theta_{\Omega_{1}}, \ldots, \theta_{\Omega_{n}}\right)$ is defined as the sheaf Euler characteristic of $\mathrm{ev}^{-1}\left(\Omega_{1} \times \cdots \times \Omega_{n}\right)$, which makes sense when this variety has positive dimension. The $K$-theoretic invariants are more challenging to compute, both because they are not enumerative, and also because they do not vanish for large degrees.

Assume for simplicity that $P$ is a maximal parabolic subgroup of $G$, so that $H_{2}(X ; \mathbb{Z})=\mathbb{Z}$. The (small) quantum $K$-theory ring $\mathrm{QK}(X)$ is a formal deformation of the Grothendieck ring $K(X)$ of algebraic vector bundles on $X$, which as a group is defined by $\mathrm{QK}(X)=K(X) \otimes_{\mathbb{Z}} \mathbb{Z}[[q]]$. The product $\theta_{u} \star \vartheta_{v}$ of two Schubert structure sheaves is defined in terms of structure constants $N_{u, v}^{w, d} \in \mathbb{Z}$ such that

$$
\theta_{u} \star \theta_{v}=\sum_{w, d \geq 0} N_{u, v}^{w, d} q^{d} \theta_{w} .
$$

In contrast to the quantum cohomology ring $\mathrm{QH}(X)$, the constants $N_{u, v}^{w, d}$ are not single Gromov-Witten invariants, but are defined as polynomial expressions of the $K$-theoretic Gromov-Witten invariants. A result of Givental asserts that $\mathrm{QK}(X)$ is an associative ring [13]. Since the $K$-theoretic Gromov-Witten invariants do not vanish for large degrees, the same might be true for the structure constants $N_{u, v}^{w, d}$, in which case the product $\theta_{u} \star \theta_{v}$ would be a power series in $q$ with infinitely many non-zero terms. When $X$ is a Grassmannian of type A, a combinatorial argument in [3] shows that this does not happen; all products in $\mathrm{QK}(X)$ are finite. In this paper we give a different geometric proof that shows more generally that all products in $\mathrm{QK}(X)$ are finite whenever $X$ is a cominuscule homogeneous space. As a consequence, the quantum $K$-theory ring $\mathrm{QK}(X)$ provides a honest deformation of $K(X)$. The class of cominuscule varieties consists of Grassmannians of type A, Lagrangian Grassmannians, maximal orthogonal Grassmannians, and quadric hypersurfaces. In addition there are two exceptional varieties of type E called the Cayley plane and the Freudenthal variety.

Let $d_{X}(n)$ be the minimal degree of a rational curve passing through $n$ general points of $X$. The numbers $d_{X}(n)$ for $n \leq 3$ have been computed explicitly in [7,9], see the table in $\S 4$ below. Our main result is the following.

Theorem 1. - Let $X$ be a cominuscule variety. Then $N_{u, v}^{w, d}=0$ for $d>d_{X}(2)$. 
Theorem 1 holds also for the structure constants of the equivariant quantum $K$-theory ring $\mathrm{QK}_{T}(X)$, see Remark 5.3. The bound on $d$ is sharp in the sense that $q^{d_{X}(2)}$ occurs in the square of a point in $\mathrm{QK}(X)$. In addition, this bound is also the best possible for the quantum cohomology ring $\mathrm{QH}(X)$ that does not depend on $u, v$, and $w$ (cf. [12]).

Our proof uses that the structure constants $N_{u, v}^{w, d}$ can be rephrased as alternating sums of certain boundary Gromov-Witten invariants. Given a sequence $\mathbf{d}=\left(d_{0}, d_{1}, \ldots, d_{r}\right)$ of effective degrees $d_{i} \in H_{2}(X ; \mathbb{Z})$ such that $d_{i}>0$ for $i>0$ and $\sum d_{i}=d$, let $M_{\mathbf{d}} \subset \bar{M}_{0,3}(X, d)$ be the closure of the locus of stable maps for which the domain is a chain of $r+1$ projective lines that map to $X$ in the degrees given by $\mathbf{d}$, the first and second marked points belong to the first projective line, and the third marked point is on the last projective line. Then any constant $N_{u, v}^{w, d}$ can be expressed as an alternating sum of sheaf Euler characteristics of varieties of the form ev ${ }^{-1}\left(\Omega_{1} \times \Omega_{2} \times \Omega_{3}\right) \cap M_{\mathbf{d}}$. We use geometric arguments to show that the terms of this sum cancel pairwise whenever $X$ is cominuscule and $d>d_{X}(2)$.

Set $Z_{\mathbf{d}}=\operatorname{ev}\left(M_{\mathbf{d}}\right) \subset X^{3}$. A key technical fact in our proof is that the general fibers of the map ev $: M_{\mathbf{d}} \rightarrow Z_{\mathbf{d}}$ are rationally connected. Notice that these fibers are boundary Gromov-Witten varieties $\mathrm{ev}^{-1}(x \times y \times z) \cap M_{\mathbf{d}}$ defined by three single points in $X$, and the result generalizes the well known fact that there is a unique rational curve of degree $d$ through three general points in the Grassmannian $\operatorname{Gr}(d, 2 d)$ [5]. In the special case when $\mathbf{d}=(d)$ and $M_{\mathbf{d}}=\bar{M}_{0,3}(X, d)$, it was shown in [3,9] that the general fibers of ev are rational; our proof uses this case as well as Graber, Harris, and Starr's criterion for rational connectivity [14].

We also need to know that $M_{\mathbf{d}}$ has rational singularities. For this we prove a relative version of the Kleiman-Bertini theorem [18] for rational singularities. This theorem implies that any boundary Gromov-Witten variety in $\overline{\mathcal{M}}_{0, n}(X, d)$ has rational singularities, for any homogeneous space $X$. The Kleiman-Bertini theorem generalizes a result of Brion asserting that rational singularities are preserved when a subvariety of a homogeneous space is intersected with a general Schubert variety [1].

Finally, if $\Omega_{1}$ and $\Omega_{2}$ are Schubert varieties in general position in a homogeneous space $X$, we prove that $\mathrm{ev}_{1}^{-1}\left(\Omega_{1}\right) \cap M_{\mathbf{d}}$ is unirational and $\mathrm{ev}_{1}^{-1}\left(\Omega_{1}\right) \cap \mathrm{ev}_{2}^{-1}\left(\Omega_{2}\right) \cap M_{\mathbf{d}}$ is either empty or unirational. In particular, we have $I_{d}\left(\theta_{\Omega_{1}}\right)=1$ and $I_{d}\left(\theta_{\Omega_{1}}, \theta_{\Omega_{2}}\right) \in\{0,1\}$. This is done by showing that any Borel-equivariant map to a Schubert variety is locally trivial over the open cell. In particular, any single evaluation map ev $\mathrm{ev}_{i}: M_{\mathbf{d}} \rightarrow X$ is locally trivial.

Our paper is organized as follows. In Section 2 we prove the Kleiman-Bertini theorem for rational singularities and give a simple criterion for an equivariant map to be locally trivial. These results are applied to (boundary) Gromov-Witten varieties of general homogeneous spaces in Section 3. Section 4 proves some useful facts about images of Gromov-Witten varieties of cominuscule spaces, among them that the general fibers of ev $: M_{\mathbf{d}} \rightarrow Z_{\mathbf{d}}$ are rationally connected. Finally, Section 5 applies these results to show that $K$-theoretic quantum products on cominuscule varieties are finite.

Parts of this work were carried out during visits to the Mathematical Sciences Research Institute (Berkeley), the Centre International de Rencontres Mathématiques (Luminy), the Hausdorff Center for Mathematics (Bonn) and the Max-Planck-Institut für Mathematik (Bonn). We thank all of these institutions for their hospitality and stimulating environments. We also benefited from helpful discussions with P. Belkale, S. Kumar, and F. Sottile. Finally, we thank the anonymous referee for a careful reading and several helpful suggestions. 


\section{A Kleiman-Bertini theorem for rational singularities}

Definition 2.1. - Let $G$ be a connected algebraic group and $X$ a $G$-variety. A splitting of the action of $G$ on $X$ is a morphism $s: U \rightarrow G$ defined on a dense open subset $U \subset X$, together with a point $x_{0} \in U$, such that $s(x) \cdot x_{0}=x$ for all $x \in U$. If a splitting exists, then we say that the action is split and that $X$ is $G$-split.

Notice that any $G$-split variety contains a dense open orbit. Recall that if $X=G / P$ is a homogeneous space defined by a semisimple complex Lie group $G$ and a parabolic subgroup $P$, then a Schubert variety in $X$ is an orbit closure for the action of a Borel subgroup of $G$. Schubert varieties are our main examples of varieties with a split action.

Proposition 2.2. - Let $G$ be a semisimple complex Lie group, $P \subset G$ a parabolic subgroup, and $X=G / P$ the corresponding homogeneous space with its natural $G$-action. Then $X$ is $G$-split. Furthermore, if $B \subset G$ is a Borel subgroup and $\Omega \subset X$ is a B-stable Schubert variety, then $\Omega$ is B-split.

Proof. - Let $\Omega \subset X$ be a $B$-stable Schubert variety, $\Omega^{\circ} \subset \Omega$ the $B$-stable open cell, and $x_{0} \in \Omega^{\circ}$ any point. According to e.g., [20, Lemma 8.3.6] we can choose a unipotent subgroup $U \subset B$ such that the map $U \rightarrow \Omega^{\circ}$ defined by $g \mapsto g \cdot x_{0}$ is an isomorphism. The inverse of this map is a splitting of the $B$-action on $\Omega$. Since $X$ is a Schubert variety, it follows that $X$ is $B$-split and consequently $G$-split.

Recall that a morphism $f: M \rightarrow X$ is a locally trivial fibration if each point $x \in X$ has an open neighborhood $U \subset X$ such that $f^{-1}(U) \cong U \times f^{-1}(x)$ and $f$ is the projection to the first factor.

Proposition 2.3. - Let $f: M \rightarrow X$ be an equivariant map of irreducible $G$-varieties. Assume that $X$ is $G$-split. Then $f$ is a locally trivial fibration over the dense open $G$-orbit in $X$, and the fibers over this orbit are irreducible.

Proof. - Let $x_{0} \in U \subset X$ and $s: U \rightarrow G$ be a splitting of the $G$-action on $X$. Then the $\operatorname{map} \varphi: U \times f^{-1}\left(x_{0}\right) \rightarrow f^{-1}(U)$ defined by $\varphi(x, y)=s(x) . y$ is an isomorphism, with inverse given by $\varphi^{-1}(m)=\left(f(m), s(f(m))^{-1} \cdot m\right)$. Since $f^{-1}(U) \cong U \times f^{-1}\left(x_{0}\right)$ is irreducible, so is $f^{-1}\left(x_{0}\right)$.

In the rest of this section, a variety means a reduced scheme of finite type over an algebraically closed field of characteristic zero. An irreducible variety $X$ has rational singularities if there exists a desingularization $\pi: \widetilde{X} \rightarrow X$ such that $\pi_{*} \theta_{\widetilde{X}}=\theta_{X}$ and $R^{i} \pi_{*} \theta_{\widetilde{X}}=0$ for all $i>0$. An arbitrary variety has rational singularities if its irreducible components have rational singularities, are disjoint, and have the same dimension. Zariski's main theorem implies that any variety with rational singularities is normal. Notice also that if $X$ and $Y$ have rational singularities, then so does $X \times Y$. The converse is a special case of the following lemma of Brion [1, Lemma 3].

Lemma 2.4 (Brion). - Let $Z$ and $S$ be varieties and let $\pi: Z \rightarrow S$ be a morphism. If $Z$ has rational singularities, then the same holds for the general fibers of $\pi$.

4 e SÉRIE - TOME $46-2013$ - No 3 
The following generalization of the Kleiman-Bertini theorem [18] was proved by Brion in [1, Lemma 2] when $p$ and $q$ are inclusions and $Y$ is a Schubert variety. We adapt his proof to our case.

TheOREM 2.5. - Let $G$ be a connected algebraic group and let $X$ be a split and transitive $G$-variety. Let $p: Y \rightarrow X$ and $q: Z \rightarrow X$ be morphisms of varieties, and assume that $Y$ and $Z$ have rational singularities. Then $g . Y \times_{X} Z$ has rational singularities for all points $g$ in a dense open subset of $G$.

Proof. - It follows from Proposition 2.3 that the map $m: G \times Y \rightarrow X$ defined by $m(g, y)=g \cdot p(y)$ is a locally trivial fibration. Set $Q=(G \times Y) \times_{X} Z$ and consider the diagram:

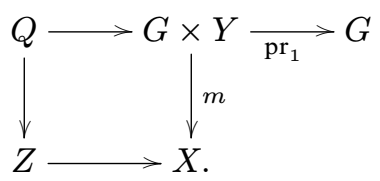

Since $G \times Y$ has rational singularities, it follows from Lemma 2.4 that $m^{-1}(x)$ has rational singularities for $x \in X$. Since $m$ is a locally trivial fibration, so is the map $Q \rightarrow Z$, hence the assumption that $Z$ has rational singularities implies that $Q$ has rational singularities. Finally, Lemma 2.4 applied to the map $Q \rightarrow G$ implies that $g . Y \times_{X} Z$ has rational singularities for all points $g$ in a dense open subset of $G$.

In the situation of Theorem 2.5, notice that if the map $p: Y \rightarrow X$ is $G$-equivariant, then the isomorphism class of $g . Y \times_{X} Z$ is independent of $g$. It follows that $Y \times_{X} Z$ has rational singularities.

\subsection{Rationality}

Before we continue, we recall some rationality properties of algebraic varieties that are required in later sections. An algebraic variety $X$ is called rational if it is birationally equivalent to a projective space $\mathbb{P}^{n}$. It is called unirational if there exists a dominant morphism $U \rightarrow X$ where $U$ is an open subset of $\mathbb{P}^{n}$; here $n$ is allowed to be greater than the dimension of $X$, but in such cases one can replace $\mathbb{P}^{n}$ with a linear subspace to obtain a generically finite map from $U$ to $X$. Finally, $X$ is said to be rationally connected if a general pair of points $(x, y) \in X \times X$ can be joined by a rational curve, i.e., both $x$ and $y$ belong to the image of some morphism $\mathbb{P}^{1} \rightarrow X$. Rational implies unirational, which in turn implies rational connectivity when $X$ is complete. Notice also that any rationally connected variety is irreducible. The relevance of these notions to our study of quantum $K$-theory originates in the fact that, if $X$ is any rationally connected non-singular projective variety, then $H^{i}\left(X, \vartheta_{X}\right)=0$ for all $i>0$ [10, Cor. 4.18(a)], hence the sheaf Euler characteristic of $X$ is equal to one. In addition, rational connectivity is one of the hypotheses needed in Proposition 5.2 below. The following result from [14] provides an important tool for proving that a variety is rationally connected.

Theorem 2.6 (Graber, Harris, Starr). - Let $f: X \rightarrow Y$ be any dominant morphism of complete irreducible complex varieties. If $Y$ and the general fiber of $f$ are rationally connected, then $X$ is rationally connected. 


\section{Geometry of Gromov-Witten varieties}

Let $X=G / P$ be a homogeneous space, where $G$ is any semisimple complex linear algebraic group and $P$ a parabolic subgroup. Given an effective class $d \in H_{2}(X ; \mathbb{Z})$ and an integer $n \geq 0$, the Kontsevich moduli space $\overline{\mathcal{M}}_{0, n}(X, d)$ parametrizes the set of all $n$-pointed stable genus-zero maps $f: C \rightarrow X$ with $f_{*}[C]=d$, and is equipped with a total evaluation map ev $=\left(\mathrm{ev}_{1}, \ldots, \mathrm{ev}_{n}\right): \overline{\mathcal{M}}_{0, n}(X, d) \rightarrow X^{n}:=X \times \cdots \times X$. A detailed construction of this space can be found in the survey [11]. The space $\overline{\mathcal{M}}_{0, n}(X, d)$ is a projective variety with rational singularities, and it was proved by Kim and Pandharipande that this variety is also rational [17].

Corollary 3.1. - Let $\Omega_{1}, \ldots, \Omega_{n} \subset X$ be Schubert varieties of $X$ in general position. Then the Gromov-Witten variety $\mathrm{ev}^{-1}\left(\Omega_{1} \times \cdots \times \Omega_{n}\right) \subset \overline{\mathcal{M}}_{0, n}(X, d)$ has rational singularities.

Proof. - Since the component-wise action of $G^{n}$ on $X^{n}$ is transitive and split, this result follows by applying Theorem 2.5 to the inclusion $\Omega_{1} \times \cdots \times \Omega_{n} \subset X^{n}$ and the evaluation map ev $: \overline{\mathcal{M}}_{0, n}(X, d) \rightarrow X^{n}$.

The following proposition will be used to show that one-point and two-point GromovWitten varieties are unirational.

Proposition 3.2. - Let $M$ be a unirational $G$-variety and let $f_{1}: M \rightarrow X$ and $f_{2}: M \rightarrow X$ be $G$-equivariant maps. Let $\Omega_{1}, \Omega_{2} \subset X$ be opposite $S$ chubert varieties.

(a) The variety $f_{1}^{-1}\left(\Omega_{1}\right) \subset M$ is unirational.

(b) The image $\widetilde{\Omega}=f_{2}\left(f_{1}^{-1}\left(\Omega_{1}\right)\right) \subset X$ is a Schubert variety in $X$, and the map $f_{2}: f_{1}^{-1}\left(\Omega_{1}\right) \rightarrow \widetilde{\Omega}$ is a locally trivial fibration over the open cell $\widetilde{\Omega}^{\circ} \subset \widetilde{\Omega}$.

(c) The intersection $f_{1}^{-1}\left(\Omega_{1}\right) \cap f_{2}^{-1}\left(\Omega_{2}\right) \subset M$ is either empty or unirational.

Proof. - It follows from Proposition 2.3 that $f_{1}$ is a locally trivial fibration, so the assumption that $M$ is unirational implies that the fibers of $f_{1}$ are unirational. Part (a) follows from this because $f_{1}: f_{1}^{-1}\left(\Omega_{1}\right) \rightarrow \Omega_{1}$ is also locally trivial. Choose opposite Borel subgroups $B, B^{\text {op }} \subset G$ such that $\Omega_{1}$ is $B$-stable and $\Omega_{2}$ is $B^{\text {op }}$-stable. Then $f_{1}^{-1}\left(\Omega_{1}\right)$ and $\widetilde{\Omega}$ are $B$-stable, and since $\widetilde{\Omega}$ is also closed in $X$ and irreducible, it is a Schubert variety. Part (b) now follows from Proposition 2.3 because $f_{2}: f_{1}^{-1}\left(\Omega_{1}\right) \rightarrow \widetilde{\Omega}$ is $B$-equivariant. Parts (a) and (b) imply that the fibers of $f_{2}: f_{1}^{-1}\left(\Omega_{1}\right) \rightarrow \widetilde{\Omega}$ over $\widetilde{\Omega}^{\circ}$ are unirational. Since $\widetilde{\Omega}$ is normal, it follows using Stein factorization that all fibers of $f_{2}: f_{1}^{-1}\left(\Omega_{1}\right) \rightarrow \widetilde{\Omega}$ are connected. If $f_{1}^{-1}\left(\Omega_{1}\right) \cap f_{2}^{-1}\left(\Omega_{2}\right) \neq \varnothing$, then the Kleiman-Bertini theorem [18, Rmk. 7] implies that this intersection is locally irreducible. Part (c) follows from this, using that the Richardson variety $\widetilde{\Omega} \cap \Omega_{2}$ is rational.

Corollary 3.3. - (a) Let $\Omega \subset X$ be a Schubert variety and $n \geq 1$. Then $\mathrm{ev}_{1}^{-1}(\Omega) \subset \bar{M}_{0, n}(X, d)$ is unirational.

(b) Let $\Omega_{1}, \Omega_{2} \subset X$ be opposite Schubert varieties and $n \geq 2$. Then the two-point GromovWitten variety $\mathrm{ev}_{1}^{-1}\left(\Omega_{1}\right) \cap \mathrm{ev}_{2}^{-1}\left(\Omega_{2}\right) \subset \overline{\mathcal{M}}_{0, n}(X, d)$ is either empty or unirational.

Proof. - This follows from parts (a) and (c) of Proposition 3.2. 
Remark 3.4. - Proposition 2.3 can also be used to prove unirationality of certain 3 -point Gromov-Witten varieties. A result of Popov [19] shows that the diagonal action of $G$ on $X^{3}$ has a dense open orbit if and only if $X$ is a cominuscule variety, and it is natural to ask if the action is also split. It turns out that a splitting can be constructed when $X$ is a Grassmann variety of type A or a maximal orthogonal Grassmannian, but no splitting exists for a Lagrangian Grassmannian. When $X^{3}$ is $G$-split, it follows from Proposition 2.3 that $\mathrm{ev}^{-1}(x, y, z) \subset \overline{\mathcal{M}}_{0,3}(X, d)$ is either empty or unirational for all points $(x, y, z)$ in the dense open $G$-orbit of $X^{3}$. This partially recovers results from [3, 9] asserting that 3-point Gromov-Witten varieties are rational for all cominuscule varieties (see Theorem 4.8 below). It is interesting to note that Lagrangian Grassmannians also required special treatment in [9].

REMARK 3.5. - Jason Starr reports that the results of [16] can be used to prove the following statement. If $P \subset G$ is a maximal parabolic subgroup and $d$ is sufficiently large, then $\mathrm{ev}^{-1}(x, y, z) \subset \overline{\mathcal{M}}_{0,3}(X, d)$ is rationally connected for all points $(x, y, z)$ in a dense open subset of $X^{3}$.

Our applications require generalizations of Corollaries 3.1 and 3.3 to Gromov-Witten varieties of stable maps with reducible domains. Let $\mathbf{d}=\left(d_{0}, d_{1}, \ldots, d_{r}\right)$ be a sequence of effective classes $d_{i} \in H_{2}(X ; \mathbb{Z})$, let $\mathbf{e}=\left(e_{0}, \ldots, e_{r}\right) \in \mathbb{N}^{r+1}$, and set $|\mathbf{d}|=\sum d_{i}$ and $|\mathbf{e}|=\sum e_{i}$. We consider stable maps $f: C \rightarrow X$ in $\bar{M}_{0,|\mathbf{e}|}(X,|\mathbf{d}|)$ defined on a chain $C$ of $r+1$ projective lines, such that the $i$-th projective line contains $e_{i}$ marked points (numbered from $1+\sum_{j<i} e_{j}$ to $\sum_{j \leq i} e_{j}$ ) and the restriction of $f$ to this component has degree $d_{i}$. To ensure that such a map is indeed stable, we demand that $e_{i} \geq 1+\delta_{i, 0}+\delta_{i, r}$ whenever $d_{i}=0$.

Let $M_{\mathbf{d}, \mathbf{e}} \subset \bar{M}_{0,|\mathbf{e}|}(X,|\mathbf{d}|)$ be the closure of the locus of all such stable maps. In the cases we are interested in, this variety can also be defined inductively as follows. If $r=0$, then $M_{d_{0}, e_{0}}=\bar{M}_{0, e_{0}}\left(X, d_{0}\right)$. Otherwise set $\mathbf{d}^{\prime}=\left(d_{0}, \ldots, d_{r-1}\right)$ and $\mathbf{e}^{\prime}=\left(e_{0}, \ldots, e_{r-2}, e_{r-1}+1\right)$, and consider the product over $X$ of the maps ev $\operatorname{ev}_{\left|\mathbf{e}^{\prime}\right|}: M_{\mathbf{d}^{\prime}, \mathbf{e}^{\prime}} \rightarrow X$ and ev $1: M_{d_{r}, e_{r}+1} \rightarrow X$. We thank the referee for pointing out that the condition $e_{0} e_{r} \neq 0$ is necessary in the following proposition, which follows from [11, Lemma 12] by induction on $r$.

Proposition 3.6. - Assume that $e_{0}>0$ and $e_{r}>0$. Then, with the above notation, we have an isomorphism

$$
M_{\mathbf{d}^{\prime}, \mathbf{e}^{\prime}} \times_{X} M_{d_{r}, e_{r}+1} \stackrel{\cong}{\longrightarrow} M_{\mathbf{d}, \mathbf{e}} .
$$

Given subvarieties $\Omega_{1}, \ldots, \Omega_{m}$ of $X$ with $m \leq|\mathbf{e}|$, define a boundary Gromov-Witten variety by $M_{\mathbf{d}, \mathbf{e}}\left(\Omega_{1}, \ldots, \Omega_{m}\right)=\bigcap_{i=1}^{m} \mathrm{ev}_{i}^{-1}\left(\Omega_{i}\right) \subset M_{\mathbf{d}, \mathbf{e}}$. The varieties $\Omega_{i}$ will often, but not always, be chosen in general position.

We also define the varieties $Z_{\mathbf{d}, \mathbf{e}}\left(\Omega_{1}, \ldots, \Omega_{m}\right)=\operatorname{ev}\left(M_{\mathbf{d}, \mathbf{e}}\left(\Omega_{1}, \ldots, \Omega_{m}\right)\right) \subset X^{|\mathbf{e}|}$ and $\Gamma_{\mathbf{d}, \mathbf{e}}\left(\Omega_{1}, \ldots, \Omega_{m}\right)=\operatorname{ev}_{|\mathbf{e}|}\left(M_{\mathbf{d}, \mathbf{e}}\left(\Omega_{1}, \ldots, \Omega_{m}\right)\right) \subset X$. If no sequence $\mathbf{e}$ is specified, we will use $\mathbf{e}=(3)$ when $r=0$ and $\mathbf{e}=(2,0, \ldots, 0,1)$ when $r>0$; this convention will only be used when $d_{i} \neq 0$ for $i>0$. For example, if $x, y \in X$ and $d \in H_{2}(X ; \mathbb{Z})$, then $\Gamma_{d}(x) \subset X$ is the union of all rational curves of degree $d$ passing through $x$, and $\Gamma_{d}(x, y)$ is the union of all rational curves of degree $d$ passing through $x$ and $y$. The variety $Z_{d, 2} \subset X \times X$ contains all pairs of points that are connected by a rational curve of degree $d$, and $Z_{d}=Z_{d, 3} \subset X \times X \times X$ consists of the triples of points connected by such a curve. 
If $\Omega \subset X$ is a $B$-stable Schubert variety, then so is $\Gamma_{\mathbf{d}}(\Omega)$ by Proposition 3.2(b) and the following result. This fact was also used in [8].

Proposition 3.7. - The variety $M_{\mathbf{d}, \mathbf{e}}$ is unirational and has rational singularities.

Proof. - By induction on $r$ we may assume that $M_{\mathbf{d}^{\prime}, \mathbf{e}^{\prime}}$ is unirational and has rational singularities. Since all maps in the Cartesian square

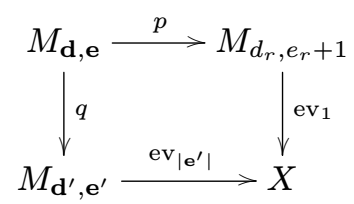

are equivariant, it follows from Theorem 2.5 that $M_{\mathbf{d}, \mathbf{e}}$ has rational singularities. Proposition 2.3 implies that ev 1 is locally trivial, and since $M_{d_{r}, e_{r}+1}$ is rational, we deduce that the fibers of $\mathrm{ev}_{1}$ are unirational. This in turn implies that $q$ is a locally trivial map with unirational fibers. Finally, since $M_{\mathbf{d}^{\prime}, \mathbf{e}^{\prime}}$ is unirational, we conclude that $M_{\mathbf{d}, \mathbf{e}}$ is unirational as well.

COROllary 3.8. - Let $\Omega_{1}, \Omega_{2}, \ldots, \Omega_{m} \subset X$ be Schubert varieties in general position, with $m \leq|\mathbf{e}|$. Then $M_{\mathbf{d}, \mathbf{e}}\left(\Omega_{1}, \ldots, \Omega_{m}\right)$ has rational singularities. Furthermore, the one-point Gromov-Witten variety $M_{\mathbf{d}, \mathbf{e}}\left(\Omega_{1}\right)$ is unirational, and the two-point Gromov-Witten variety $M_{\mathbf{d}, \mathbf{e}}\left(\Omega_{1}, \Omega_{2}\right)$ is either empty or unirational.

Let $d \in H_{2}(X ; \mathbb{Z})$ be an effective class and let $\pi_{1}, \pi_{2}: Z_{d, 2} \rightarrow X$ denote the projections. Define the variety

$$
Z_{d, 2}^{*}=Z_{d, 2} \backslash \bigcup_{d^{\prime}} Z_{d^{\prime}, 2}
$$

where the union is over all degrees $d^{\prime} \in H_{2}(X ; \mathbb{Z})$ for which $Z_{d^{\prime}, 2} \varsubsetneqq Z_{d, 2}$. This is a $G$-stable dense open subset of $Z_{d, 2}$ because $Z_{d, 2}=\operatorname{ev}\left(M_{d, 2}\right)$ is irreducible. For $x \in X$ we also set $\Gamma_{d}^{*}(x)=\pi_{2}\left(\pi_{1}^{-1}(x) \cap Z_{d, 2}^{*}\right)$, a dense open subset of $\Gamma_{d}(x)$.

Lemma 3.9. - Let $d \in H_{2}(X ; \mathbb{Z})$ be an effective class and $\Omega \subset X$ a Schubert variety.

(a) The variety $Z_{d, 2}$ is rational and has rational singularities.

(b) The intersection $\Omega \cap \Gamma_{d}(z)$ is unirational and has rational singularities for all points $z$ in the open cell $\Gamma_{d}(\Omega)^{\circ} \subset \Gamma_{d}(\Omega)$.

(c) Let $\Omega^{*} \subset \Omega$ be any dense open subset. Then $\Omega^{*} \cap \Gamma_{d}^{*}(z) \neq \varnothing$ for all points $z$ in a dense open subset of $\Gamma_{d}(\Omega)$.

Proof. - It follows from Proposition 2.3 that the projection $\pi_{1}: Z_{d, 2} \rightarrow X$ is a locally trivial fibration. Since each fiber $\pi_{1}^{-1}(x) \cong \Gamma_{d}(x)$ is a Schubert variety in $X$, we deduce that $\pi_{1}^{-1}(\Omega)$ is rational and has rational singularities. Part (a) follows as a special case of this. Since Proposition 3.2(b) implies that $\pi_{2}: \pi_{1}^{-1}(\Omega) \rightarrow \Gamma_{d}(\Omega)$ is a locally trivial fibration over $\Gamma_{d}(\Omega)^{\circ}$, it follows that $\pi_{1}^{-1}(\Omega) \cap \pi_{2}^{-1}(z) \cong \Omega \cap \Gamma_{d}(z)$ is unirational for all $z \in \Gamma_{d}(\Omega)^{\circ}$, and Lemma 2.4 implies that $\Omega \cap \Gamma_{d}(z)$ has rational singularities. This proves (b). Since $\pi_{1}\left(Z_{d, 2}^{*}\right)=X$ and $\pi_{1}^{-1}(\Omega)$ is irreducible, we deduce that $U=\pi_{1}^{-1}\left(\Omega^{*}\right) \cap Z_{d, 2}^{*}$ is a dense open subset of $\pi_{1}^{-1}(\Omega)$. Part (c) follows from this because $\pi_{2}(U)$ contains a dense open subset of $\Gamma_{d}(\Omega)$, and $\Omega^{*} \cap \Gamma_{d}^{*}(z) \cong U \cap \pi_{2}^{-1}(z) \neq \varnothing$ for all $z \in \pi_{2}(U)$. 


\section{Gromov-Witten varieties of cominuscule spaces}

Let $X=G / P$ be a homogeneous space, where $G$ is a simple complex linear algebraic group and $P$ is a maximal parabolic subgroup. In the remainder of this paper we require more precise notation for the Schubert varieties in $X$, which we now introduce. Fix a maximal torus $T$ and a Borel subgroup $B$ such that $T \subset B \subset P \subset G$, and let $R$ be the associated root system, with positive roots $R^{+} \subset R$ and simple roots $\Delta \subset R^{+}$. Let $W=N_{G}(T) / T$ be the Weyl group of $G$ and $W_{P}=N_{P}(T) / T \subset W$ the Weyl group of $P$. The subgroup $P$ corresponds to a simple root $\alpha \in \Delta$, such that $W_{P}$ is generated by all simple reflections except $s_{\alpha}$. The variety $X$ is called cominuscule if $\alpha$ is a cominuscule simple root, i.e., when the highest root of $R$ is expressed as a linear combination of simple roots, the coefficient of $\alpha$ is one. The collection of cominuscule varieties include the type A Grassmannians $\operatorname{Gr}(m, n)$, Lagrangian Grassmannians LG $(n, 2 n)$, maximal orthogonal Grassmannians OG $(n, 2 n)$, quadric hypersurfaces $Q^{n} \subset \mathbb{P}^{n+1}$, as well as two exceptional varieties called the Cayley Plane $\left(E_{6} / P_{6}\right)$ and the Freudenthal variety $\left(E_{7} / P_{7}\right)$. We will assume that $X$ is cominuscule in the following.

Each element $u \in W$ defines a $T$-fixed point $u . P \in X$ and a Schubert variety $X(u)=$ $\overline{B u . P} \subset X$. Both $u . P$ and $X(u)$ depend only on the coset of $u$ in $W / W_{P}$. Let $W^{P} \subset W$ be the set of minimal length representatives for the cosets in $W / W_{P}$. Then $W^{P}$ is in oneto-one correspondence with the set of $T$-fixed points in $X$ as well as the set of $B$-stable Schubert varieties in $X$, and for $u \in W^{P}$ we have $\operatorname{dim} X(u)=\ell(u)$. We will identify $H_{2}(X ; \mathbb{Z})=\mathbb{Z}\left[X\left(s_{\alpha}\right)\right]$ with the group of integers. The degree of a curve $C \subset X$ is the integer $d \in \mathbb{N}$ for which $[C]=d\left[X\left(s_{\alpha}\right)\right]$.

Given two points $x, y \in X$, we let $d(x, y)$ denote the smallest degree of a rational curve containing $x$ and $y$ [22]. Equivalently, $d(x, y)$ is minimal with the property that $(x, y) \in$ $Z_{d(x, y), 2}$. For any $n \in \mathbb{N}$ we also let $d_{X}(n)$ be the smallest degree for which any collection of $n$ points in $X$ is contained in a connected rational curve of degree $d_{X}(n)$, i.e., $d_{X}(n)$ is minimal such that $Z_{d_{X}(n), n}=X^{n}$. Notice that $d_{X}(2)=\max \{d(x, y) \mid x, y \in X\}$, and it follows from [12] that $d_{X}(2)$ is the smallest degree of the quantum parameter $q$ that occurs in the square of a point in the small quantum ring $\mathrm{QH}(X)$. Furthermore, for any degree $d \in \mathbb{N}$ we have $Z_{d, 2}^{*}=\left\{(x, y) \in X^{2} \mid d(x, y)=d^{\prime}\right\}$ and $\Gamma_{d}^{*}(x)=\left\{y \in X \mid d(x, y)=d^{\prime}\right\}$, where $d^{\prime}=\min \left(d, d_{X}(2)\right)$. The numbers $d_{X}(2)$ and $d_{X}(3)$ were computed in [7, Prop. 18] and [9, Prop. 3.4]. We reproduce these numbers in the following table, correcting some typos from [9]. The proof of Proposition 4.5 will also provide an argument for the values of most of these numbers.

\begin{tabular}{cccc}
\hline$X$ & $\operatorname{dim}(X)$ & $d_{X}(2)$ & $d_{X}(3)$ \\
\hline $\operatorname{Gr}(m, m+k)$ & $m k$ & $\min (m, k)$ & $\min (2 m, 2 k, \max (m, k))$ \\
$\mathrm{LG}(n, 2 n)$ & $\frac{n(n+1)}{2}$ & $n$ & $n$ \\
$\mathrm{OG}(n, 2 n)$ & $\frac{n(n-1)}{2}$ & $\left\lfloor\frac{n}{2}\right\rfloor$ & $\left\lceil\frac{n}{2}\right\rceil$ \\
$Q^{n}$ & $n$ & 2 & 2 \\
$E_{6} / P_{6}$ & 18 & 2 & 4 \\
$E_{7} / P_{7}$ & 27 & 3 & 3 \\
\hline
\end{tabular}


We require the following proposition, which combines parts of Prop. 18 and Lemma 21 from [7]. Notice that part (c) implies that $d_{X}(2)$ is equal to the number of occurrences of $s_{\alpha}$ in a reduced word for the element $u \in W^{P}$ for which $X(u)=X$.

Proposition 4.1 ([7]). - Let $X=G / P$ be a cominuscule variety.

(a) The diagonal action of $G$ on $Z_{d, 2}^{*}$ is transitive for each $d \in\left[0, d_{X}(2)\right]$.

(b) Let $x, y \in X$ and set $d=d(x, y)$. Then the stabilizer in $G$ of the subvariety $\Gamma_{d}(x, y) \subset X$ is a parabolic subgroup of $G$ that acts transitively on $\Gamma_{d}(x, y)$.

(c) Let $u \in W^{P}$. Then $d(1 . P, u . P)$ is the number of occurrences of $s_{\alpha}$ in any reduced expression for $u$.

Proposition 4.1 is the foundation of the following construction from [7]. Fix a degree $d \in\left[0, d_{X}(2)\right]$ and let $Y_{d}$ be the set of all subvarieties $\Gamma_{d}(x, y)$ of $X$ for which $d(x, y)=d$. The group $G$ acts on $Y_{d}$ by translation, and by parts (a) and (b) of Proposition 4.1 we can identify $Y_{d}$ with a projective homogeneous space for $G$. The points of the variety $Y_{d}$ provide a generalization to cominuscule varieties of the kernel-span pairs of curves in classical Grassmannians [4, 5]. For example, when $X=\operatorname{Gr}(m, n)$ is a Grassmannian of type $\mathrm{A}$, the space $Y_{d}$ is the two-step flag variety $\mathrm{Fl}(m-d, m+d ; n)$ of kernel-span pairs of expected dimension. Notice that the homogeneous space $Y_{d}$ contains a unique $B$-fixed point, which implies that there is a unique $B$-stable Schubert variety in $X$ of the form $\Gamma_{d}(x, y)$. We denote this non-singular Schubert variety by $X_{d}$.

Lemma 4.2. - Let $x, y \in X$. Then there exists a chain of $d(x, y)$ rational curves of degree 1 through $x$ and $y$.

Proof. - We may assume that $x=1 . P$ and $y=u . P$ are $T$-fixed points, with $u \in W^{P}$. Let $u=s_{\beta_{1}} s_{\beta_{2}} \cdots s_{\beta_{\ell}}$ be a reduced expression for $u$, and let $j_{1}<j_{2}<\cdots<j_{d(x, y)}$ be the indices for which $\beta_{j_{i}}=\alpha$. Set $u_{0}=1$ and $u_{i}=s_{\beta_{1}} s_{\beta_{2}} \cdots s_{\beta_{j_{i}}}$ for each $i \in[1, d(x, y)]$. Then $u_{0} \cdot P=x$, and since $u \in W^{P}$, we must have $u=u_{d(x, y)}$, so $u_{d(x, y)} \cdot P=y$. Finally notice that $d\left(u_{i-1} . P, u_{i} . P\right)=d\left(1 . P, u_{i-1}^{-1} u_{i} . P\right)=1$, so $u_{i-1} . P$ and $u_{i} . P$ can be joined by a line in $X$.

REMARK 4.3. - The assumption that $X$ is cominuscule is necessary in Lemma 4.2. For example, if $X=\mathrm{OG}(2,7)$ is the orthogonal Grassmannian of 2-dimensional isotropic subspaces in $\mathbb{C}^{7}$ equipped with a non-degenerate symmetric bilinear form, then two general points in $X$ are joined by an irreducible curve of degree 2, but not by a union of two lines. The same is true if $X$ is any adjoint variety [8].

It follows from Lemma 4.2 that if $\mathbf{d}=\left(d_{0}, \ldots, d_{r}\right) \in \mathbb{N}^{r+1}$ is any sequence with $d_{i}>0$ for $i>0$, and $\Omega \subset X$ is any closed subvariety, then $\Gamma_{\mathbf{d}}(\Omega)=\Gamma_{|\mathbf{d}|}(\Omega)$. The following result shows how to find the degree 1 neighborhood of a Schubert variety in $X$. Let $w_{P}$ denote the unique longest element in $W_{P}$.

Lemma 4.4. - Let $u \in W^{P}$ be such that $X(u) \neq X$. Then $\Gamma_{1}(X(u))=X\left(u w_{P} s_{\alpha}\right)$. 
Proof. - Notice that $\Gamma_{1}(X(u))$ is a $B$-stable Schubert variety in $X$. The inclusion $X\left(u w_{P} s_{\alpha}\right) \subset \Gamma_{1}(X(u))$ follows because $d\left(u . P, u w_{P} s_{\alpha} \cdot P\right)=d\left(1 . P, s_{\alpha} \cdot P\right)=1$. To prove the opposite inclusion it is enough to show that any $T$-fixed point $w \cdot P \in \Gamma_{1}(X(u))$ is contained in $X\left(u w_{P} s_{\alpha}\right)$. Since $T$ acts on the projective variety of all degree one curves from $w . P$ to $X(u)$, there exists a $T$-stable curve of this kind, hence we have $d(w \cdot P, v \cdot P)=1$ for some fixed point $v . P \in X(u)$. We may assume that $v, w \in W^{P}$, so that $v \leq u$. Proposition 4.1(c) implies that we can write $v^{-1} w=x s_{\alpha} y$ where $x, y \in W_{P}$. Since the assumption $X(u) \neq X$ implies that $u w_{P} \leq u w_{P} s_{\alpha}$, we obtain $w y^{-1}=v x s_{\alpha} \leq u w_{P} s_{\alpha}$, so $w \cdot P \in X\left(u w_{P} s_{\alpha}\right)$ as required.

Proposition 4.5. - Let $d \in\left[0, d_{X}(2)\right]$. Then we have $\Gamma_{d_{X}(3)-d}\left(X_{d}\right)=X$.

Proof. - We check the truth of this statement case by case. Assume first that $X=\operatorname{Gr}(m, n)$ is the type A Grassmannian of all $m$-dimensional subspaces in $\mathbb{C}^{n}$. Set $k=n-m$. Since $\operatorname{Gr}(m, n) \cong \operatorname{Gr}(k, n)$, we may assume that $m \leq k$. It follows from [4, Lemma 1] and [5, Prop. 1] that for $x, y \in X$ we have $d(x, y)=\operatorname{dim}(x+y)-m$, where $x+y=\operatorname{Span}(x, y) \subset \mathbb{C}^{n}$. It follows that $d_{X}(2)=m$. We furthermore have $X_{d}=\operatorname{Gr}(d, B / A)=\{x \in X \mid A \subset x \subset B\}$ for some $(A, B) \in \operatorname{Fl}(m-d, m+d ; n)$. We claim that $\Gamma_{k-d}\left(X_{d}\right)=X$. Let $y \in X$ be any point and notice that $\operatorname{dim}(B \cap y) \geq$ $2 m+d-n=m+d-k$. Since $m+d-k \leq d$, there exists a point $x \in X_{d}$ such that $\operatorname{dim}(x \cap y) \geq m+d-k$, or equivalently $d(x, y) \leq k-d$, as required. We finally prove that $d_{X}(3)=\min (k, 2 m)$. The inequality $d_{X}(3) \geq \min (k, 2 m)$ follows from [4, Lemma 1], and the opposite inequality follows from the claim and the observation that $\Gamma_{2 m-d}\left(X_{d}\right) \supset \Gamma_{m}$ (point) $=X$. The identity $\Gamma_{d_{X}(3)-d}\left(X_{d}\right)=X$ follows.

Next assume that $X=\mathrm{LG}(n, 2 n)$ is the Lagrangian Grassmannian of maximal isotropic subspaces of a symplectic vector space $\mathbb{C}^{2 n}$. For $x, y \in X$ we have $d(x, y)=\operatorname{dim}(x+y)-n$, which implies that $d_{X}(2)=n$, and $X_{d}=\operatorname{LG}\left(A^{\perp} / A\right) \subset X$ for some isotropic $A \subset \mathbb{C}^{2 n}$ with $\operatorname{dim}(A)=n-d$. In particular, we have $X_{n}=X$, so $d_{X}(3)=n$. Let $y \in X$ be any point and set $x=\left(y \cap A^{\perp}\right)+A$. Then $x \subset \mathbb{C}^{2 n}$ is isotropic. Write $\operatorname{dim}(y+x)=\operatorname{dim}(y+A)=n+t$. Then $\operatorname{dim}\left(y \cap A^{\perp}\right)=\operatorname{dim}\left((y+A)^{\perp}\right)=n-t$ and $\operatorname{dim}(y \cap A)=n-t-d$. It follows that $\operatorname{dim}(x)=(n-t)+(n-d)-(n-t-d)=n$, so $x \in X_{d}$ and $d(x, y)=t \leq n-d$, as required.

Let $X=\mathrm{OG}(n, 2 n)$ be an orthogonal Grassmannian. Given an orthogonal form on $\mathbb{C}^{2 n}$ and a fixed maximal isotropic subspace $x_{0} \subset \mathbb{C}^{2 n}$, this space consists of all maximal isotropic subspaces $x \subset \mathbb{C}^{2 n}$ such that $\operatorname{dim}\left(x+x_{0}\right)-n$ is even. We have $d(x, y)=\frac{1}{2}(\operatorname{dim}(x+y)-n)$ for $x, y \in X$, hence $d_{X}(2)=\left\lfloor\frac{n}{2}\right\rfloor$, and $X_{d}=\mathrm{OG}\left(2 d, A^{\perp} / A\right) \subset X$ for some isotropic subspace $A \subset \mathbb{C}^{2 n}$ of dimension $n-2 d$. We claim that $d_{X}(3)=\left\lceil\frac{n}{2}\right\rceil$ and $\Gamma_{d_{X}(3)-d}\left(X_{d}\right)=X$. Given any point $y \in X$, set $x=\left(y \cap A^{\perp}\right)+A$ and write $\operatorname{dim}(x+y)=n+t$. Then $\operatorname{dim}(x)=n$ and $t \leq n-2 d$. If $t$ is even, then $x \in X_{d}$ and $d(x, y)=\frac{t}{2} \leq\left\lfloor\frac{n}{2}\right\rfloor-d$. Otherwise we can find a point $z \in X_{d}$ such that $\operatorname{dim}(x+z)=n+1$, and this implies that $d(z, y)=\frac{t+1}{2} \leq\left\lceil\frac{n}{2}\right\rceil-d$, proving the claim.

Let $X=Q^{n} \subset \mathbb{P}\left(\mathbb{C}^{n+2}\right)$ be a quadric hypersurface consisting of all isotropic lines through the origin in the vector space $\mathbb{C}^{n+2}$ equipped with an orthogonal form. Then [7, Prop. 18] shows that $d_{X}(2)=2$ and $X_{2}=X$. It follows that $d_{X}(3)=2$. We must show that $\Gamma_{1}\left(X_{1}\right)=X$. We have $X_{1}=\mathbb{P}(V)$ for some 2-dimensional isotropic subspace $V \subset \mathbb{C}^{n+2}$. 
Given any point $y \in X$, choose a 1-dimensional subspace $x \subset V \cap y^{\perp}$. Then $x \in X_{1}$, and since $x$ and $y$ are joined by the line $\mathbb{P}(x+y) \subset Q^{n}$ we have $d(x, y)=1$, as required.

Let $X=E_{7} / P_{7}$ be the Freudenthal variety. In other words, $G$ has type $E_{7}$ and $\alpha$ is the 7-th simple root of the Dynkin diagram:

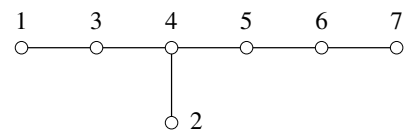

According to [7, Prop. 18] we have $d_{X}(2)=3$ and $X_{3}=X$, which implies that $d_{X}(3)=3$. The description of the varieties $X_{d}$ in terms of quivers given in [7] also reveals that $X_{2}=X\left(s_{7} s_{6} s_{5} s_{4} s_{2} s_{3} s_{4} s_{5} s_{6} s_{7}\right)$. (Alternatively, the variety $X_{2} \cong Q^{10}$ can be identified as the unique non-singular Schubert variety in $X$ of dimension 10.) By Lemma 4.4 we now obtain $\Gamma_{1}\left(X_{2}\right)=X$. We also have $X_{1}=X\left(s_{7}\right), \Gamma_{1}\left(X_{1}\right)=$ $X\left(s_{1} s_{3} s_{4} s_{2} s_{5} s_{4} s_{3} s_{1} s_{7} s_{6} s_{5} s_{4} s_{2} s_{3} s_{4} s_{5} s_{6} s_{7}\right)$, and $\Gamma_{1}\left(\Gamma_{1}\left(X_{1}\right)\right)=X$.

Finally, let $X=E_{6} / P_{6}$ be the Cayley plane, i.e., $G$ has type $E_{6}$ and $\alpha$ is the 6-th simple root of the Dynkin diagram obtained by discarding node 7 in the above diagram. By [7, Prop. 18] we have $d_{X}(2)=2$ and [9, Lemma 2.14] shows that $d_{X}(3)=4$. We obtain $d_{X}(3)-d \geq 2$, so $\Gamma_{d_{X}(3)-d}\left(X_{d}\right) \supset \Gamma_{2}$ (point) $=X$.

Corollary 4.6. - Let $x, y \in X$ and let $d \geq d(x, y)$. Then we have $\Gamma_{d}(x, y)=$ $\Gamma_{d-d(x, y)}\left(\Gamma_{d(x, y)}(x, y)\right)$. In particular, $\Gamma_{d}(x, y)$ is a Schubert variety in $X$.

Proof. - Let $z \in \Gamma_{d}(x, y)$. We must show that $z \in \Gamma_{d-d_{0}}\left(\Gamma_{d_{0}}(x, y)\right)$, where $d_{0}=d(x, y)$. If $d \geq d_{X}(3)$, then this follows from Proposition 4.5. On the other hand, if $d \leq d_{X}(2)$, then [7, Prop. 19] implies that $x, y, z$ are contained in a translate of $X_{d}$. It follows from [7, Prop. 18] that $X_{d}$ is a cominuscule variety, and using [7, Fact 20] we obtain $d_{X_{d}}(3)=d$. It now follows from Proposition 4.5 applied to $X_{d}$ that $z \in \Gamma_{d-d_{0}}\left(\Gamma_{d_{0}}(x, y)\right)$. Finally, if $d_{X}(2)<d<d_{X}(3)$, then $X$ is a Grassmannian of type A or the Cayley plane $E_{6} / P_{6}$. We consider these cases in turn.

If $X=\operatorname{Gr}(m, n)$ is a Grassmannian of type A, then [4, Lemma 1] implies $x, y, z \in X^{\prime}:=$ $\operatorname{Gr}(m-a, B / A)$ for some subspaces $A \subset B \subset \mathbb{C}^{n}$ such that $a:=\operatorname{dim}(A) \geq m-d$ and $\operatorname{dim}(B) \leq m+d$. Since $d_{X^{\prime}}(3) \leq d$ we deduce that $z \in \Gamma_{d-d_{0}}\left(\Gamma_{d_{0}}(x, y)\right)$ by applying Proposition 4.5 to $X^{\prime}$.

Finally, assume that $X=E_{6} / P_{6}$ is the Cayley plane, in which case we have $d=3$. Since $\Gamma_{2}\left(X_{1}\right)=X$, we may also assume that $d_{0}=2$ and $\Gamma_{2}(x, y)=X_{2}$. With the notation from the proof of Proposition 4.5 we have $X_{2}=X\left(s_{6} s_{5} s_{4} s_{2} s_{3} s_{4} s_{5} s_{6}\right)$, the only non-singular Schubert variety of dimension 8, and Lemma 4.4 implies that $\Gamma_{1}\left(X_{2}\right)$ is the unique Schubert divisor in $X$. Since $d_{X}(3)=4$, we also have $\Gamma_{1}\left(X_{2}\right) \subset \Gamma_{3}(x, y) \subsetneq X$. We conclude that $\Gamma_{1}\left(X_{2}\right)=\Gamma_{3}(x, y)$ as $\Gamma_{3}(x, y)$ is irreducible.

Corollary 4.7. - Let $\mathbf{d}=\left(d_{0}, d_{1}, \ldots, d_{r}\right)$ be a sequence with $d_{i}>0$ for $i>0$. Then $Z_{\mathbf{d}}=\left\{(x, y, z) \in Z_{d_{0}, 2} \times X \mid z \in \Gamma_{|\mathbf{d}|}(x, y)\right\}$.

Proof. - By definition we have $Z_{\mathbf{d}}=\left\{(x, y, z) \in X^{3} \mid z \in \Gamma_{\mathbf{d}}(x, y)\right\}$. The corollary is true because $\Gamma_{\mathbf{d}}(x, y) \neq \varnothing$ if and only if $(x, y) \in Z_{d_{0}, 2}$, in which case we have $\Gamma_{\mathbf{d}}(x, y)=\Gamma_{|\mathbf{d}|}(x, y)$ by Corollary 4.6 and Lemma 4.2. 
We need the following rationality property of Gromov-Witten varieties defined by triples of points, which in some cases are in special position [9, Thm. 0.2]. Notice that when $X$ is a Grassmannian of type A, this follows from [3, Thm. 2.1].

Theorem 4.8 ([9]). - Let $X=G / P$ be a cominuscule variety and let $d \in \mathbb{N}$. Then the variety $M_{d, 3}(x, y, z) \subset \bar{M}_{0,3}(X, d)$ is rational for all points $(x, y, z)$ in a dense open subset of $Z_{d, 3} \subset X^{3}$.

TheOrem 4.9. - Let $X=G / P$ be cominuscule and $\mathbf{d}=\left(d_{0}, \ldots, d_{r}\right)$ any sequence with $d_{i}>0$ for $i>0$. Then $M_{\mathbf{d}}(x, y, z)$ is rationally connected for all points $(x, y, z)$ in a dense open subset of $Z_{\mathbf{d}} \subset X^{3}$.

Proof. - The result follows from Theorem 4.8 when $r=0$, so assume that $r>0$. By induction on $r$ we may assume that $M_{\mathbf{d}^{\prime}}(x, y, t)$ is rationally connected for all points $(x, y, t)$ in a dense open subset of $\mathcal{Z}_{\mathbf{d}^{\prime}}$, where $\mathbf{d}^{\prime}=\left(d_{0}, \ldots, d_{r-1}\right)$. Let $(x, y) \in \mathcal{Z}_{d_{0}, 2}^{*}$ and set $\Omega=\Gamma_{\mathbf{d}^{\prime}}(x, y)$. Since $G$ acts transitively on $Z_{d_{0}, 2}^{*}$ by Proposition 4.1(a), there exists a dense open subset $\Omega^{*} \subset \Omega$ such that $M_{\mathbf{d}^{\prime}}(x, y, t)$ is rationally connected for all $t \in \Omega^{*}$. By Lemma 3.9 there exists a dense open subset $\Gamma^{*} \subset \Gamma_{\mathbf{d}}(x, y)=\Gamma_{d_{r}}(\Omega)$ such that $\Omega^{*} \cap \Gamma_{d_{r}}^{*}(z) \neq \varnothing$ and $\Omega \cap \Gamma_{d_{r}}(z)$ is unirational and normal for all $z \in \Gamma^{*}$.

By replacing $\Gamma^{*}$ with a smaller set, we may also assume that $M_{\mathbf{d}}(x, y, z)$ is locally irreducible for all $z \in \Gamma^{*}$. Indeed, since $M_{\mathbf{d}}(x)$ is unirational by Corollary 3.8, and Proposition 3.2(b) implies that $\mathrm{ev}_{2}: M_{\mathbf{d}}(x) \rightarrow \Gamma_{d_{0}}(x)$ is locally trivial over $\Gamma_{d_{0}}^{*}(x)$, it follows that $M_{\mathbf{d}}(x, y)$ is unirational. Since $\Gamma_{\mathbf{d}}(x, y)$ is a Schubert variety by Corollary 4.6 and $\mathrm{ev}_{3}: M_{\mathbf{d}}(x, y) \rightarrow \Gamma_{\mathbf{d}}(x, y)$ is surjective, the Kleiman-Bertini theorem [18, Rmk. 7] applied to the Borel action on the open cell $\Gamma_{\mathbf{d}}(x, y)^{\circ}$ shows that $M_{\mathbf{d}}(x, y, z)$ is locally irreducible for all points $z$ in a dense open subset of $\Gamma_{\mathbf{d}}(x, y)$.

We claim that $M_{\mathbf{d}}(x, y, z)$ is rationally connected for all $z \in \Gamma^{*}$. The space $M_{\mathbf{d}}$ is the product of the maps ev $\mathrm{e}_{3}: M_{\mathrm{d}^{\prime}} \rightarrow X$ and $\mathrm{ev}_{1}: M_{d_{r}, 2} \rightarrow X$. Let $f: M_{\mathbf{d}} \rightarrow X$ be the morphism defined by the product. This map restricts to a surjective morphism $M_{\mathbf{d}}(x, y, z) \rightarrow \Omega \cap \Gamma_{d_{r}}(z)$, whose fibers are given by $f^{-1}(t) \cap M_{\mathbf{d}}(x, y, z)=M_{\mathbf{d}^{\prime}}(x, y, t) \times$ $M_{d_{r}, 2}(t, z)$. Since $M_{d_{r}, 2}(z)$ is unirational and Proposition 3.2(b) implies that the map $\mathrm{ev}_{2}: M_{d_{r}, 2}(z) \rightarrow \Gamma_{d_{r}}(z)$ is locally trivial over $\Gamma_{d_{r}}^{*}(z)$, it follows that $M_{d_{r}, 2}(t, z)$ is unirational for all $t \in \Gamma_{d_{r}}^{*}(z)$. We deduce that $f^{-1}(t) \cap M_{\mathbf{d}}(x, y, z)$ is rationally connected for all $t \in \Omega^{*} \cap \Gamma_{d_{r}}^{*}(z)$. By using the Stein factorization of the map $M_{\mathbf{d}}(x, y, z) \rightarrow \Omega \cap \Gamma_{d_{r}}(z)$ and the fact that $\Omega \cap \Gamma_{d_{r}}(z)$ is normal, it follows from Zariski's main theorem [15, III.11.4] that $M_{\mathbf{d}}(x, y, z)$ is connected and therefore irreducible. The claim now follows from Theorem 2.6.

Define a morphism $\rho: G \times \Gamma_{\mathbf{d}}(x, y) \rightarrow Z_{\mathbf{d}}$ by $\rho(g, z)=(g . x, g . y, g . z)$. It follows from Proposition 4.1(a) that the image of $\rho$ contains $Z_{\mathbf{d}} \cap\left(Z_{d_{0}, 2}^{*} \times X\right)$, which is a dense open subset of $\mathcal{Z}_{\mathbf{d}}$. This implies that $\rho\left(G \times \Gamma^{*}\right)$ contains a dense open subset of $\mathcal{Z}_{\mathbf{d}}$, which completes the proof of the theorem. 


\section{Quantum $K$-theory of cominuscule varieties}

Let $X=G / P$ be a cominuscule variety and let $K(X)$ denote its Grothendieck ring. A short summary of the properties of this ring can be found in [6, §3], while many more details can be found in [2]. Each element $w \in W^{P}$ defines a Schubert class $\theta_{w}=\left[\theta_{X\left(w_{0} w\right)}\right] \in K(X)$, where $w_{0} \in W$ is the longest element, and these classes form a $\mathbb{Z}$-basis for $K(X)$. The dual Schubert classes $\theta_{w}^{\vee} \in K(X)$ are defined by $\chi_{X}\left(\theta_{u} \cdot \theta_{v}^{\vee}\right)=\delta_{u, v}$ for $u, v \in W^{P}$, where $\chi_{X}: K(X) \rightarrow \mathbb{Z}$ is the sheaf Euler characteristic map.

Given classes $\alpha_{1}, \ldots, \alpha_{n} \in K(X)$, we set $\alpha_{1} \otimes \cdots \otimes \alpha_{n}=\prod_{i=1}^{n} \pi_{i}^{*}\left(\alpha_{i}\right) \in K\left(X^{n}\right)$, where $\pi_{i}: X^{n} \rightarrow X$ is the $i$-th projection. Together with a degree $d \in \mathbb{N}$, these classes define the $K$-theoretic Gromov-Witten invariant

$$
I_{d}\left(\alpha_{1}, \ldots, \alpha_{n}\right)=\chi_{M_{d, n}}\left(\mathrm{ev}^{*}\left(\alpha_{1} \otimes \cdots \otimes \alpha_{n}\right)\right) .
$$

The quantum $K$-theory ring of $X$ is an algebra over $\mathbb{Z}[[q]]$, which as a $\mathbb{Z}[[q]]$-module is given by $\mathrm{QK}(X)=K(X) \otimes_{\mathbb{Z}} \mathbb{Z}[[q]]$. The multiplicative structure is defined by

$$
\theta_{u} \star \theta_{v}=\sum_{w, d} N_{u, v}^{w, d} q^{d} \theta_{w}
$$

where the sum is over all $w \in W^{P}$ and $d \in \mathbb{N}$. The structure constants $N_{u, v}^{w, d}$ are defined by

$$
N_{u, v}^{w, d}=\sum_{\mathbf{d}=\left(d_{0}, \ldots, d_{r}\right), \kappa_{1}, \ldots, \kappa_{r}}(-1)^{r} I_{d_{0}}\left(\theta_{u}, \theta_{v}, \theta_{\kappa_{1}}^{\vee}\right) \prod_{i=1}^{r} I_{d_{i}}\left(\theta_{\kappa_{i}}, \theta_{\kappa_{i+1}}^{\vee}\right),
$$

the sum over all sequences $\mathbf{d}=\left(d_{0}, \ldots, d_{r}\right)$ with $|\mathbf{d}|=d$ and $d_{i}>0$ for $i>0$, and all elements $\kappa_{1}, \ldots, \kappa_{r} \in W^{P}$. Notice that the sign $(-1)^{r}$ and the number of elements $\kappa_{i}$ depend on the length of $\mathbf{d}$, and we write $\kappa_{r+1}=w$. A theorem of Givental [13] states that $\mathrm{QK}(X)$ is an associative ring.

In this section we prove that any product of Schubert classes in $\mathrm{QK}(X)$ has only finitely many non-zero terms. We start by observing that each structure constant $N_{u, v}^{w, d}$ can also be expressed as an alternating sum of Euler characteristics computed on the spaces $M_{\mathbf{d}}$. The following lemma generalizes to any homogeneous space with the same proof.

Lemma 5.1. - Let $u, v, w \in W^{P}$ and let $\mathbf{d}=\left(d_{0}, d_{1}, \ldots, d_{r}\right)$ be any sequence such that $d_{i}>0$ for $i>0$. Then

$$
\chi_{M_{\mathbf{d}}}\left(\mathrm{ev}^{*}\left(\theta_{u} \otimes \theta_{v} \otimes \theta_{w}^{\vee}\right)\right)=\sum_{\kappa_{1}, \ldots, \kappa_{r}} I_{d_{0}}\left(\theta_{u}, \theta_{v}, \theta_{\kappa_{1}}^{\vee}\right) \prod_{i=1}^{r} I_{d_{i}}\left(\theta_{\kappa_{i}}, \theta_{\kappa_{i+1}}^{\vee}\right)
$$

where the sum is over all $\kappa_{1}, \ldots, \kappa_{r} \in W^{P}$ and we set $\kappa_{r+1}=w$.

Proof. - We may assume that $r>0$. Set $\mathbf{d}^{\prime}=\left(d_{0}, \ldots, d_{r-1}\right)$. It is enough to show that

$$
\chi_{M_{\mathbf{d}}}\left(\operatorname{ev}^{*}\left(\theta_{u} \otimes \theta_{v} \otimes \theta_{w}^{\vee}\right)\right)=\sum_{\kappa \in W^{P}} \chi_{M_{\mathbf{d}^{\prime}}}\left(\operatorname{ev}^{*}\left(\theta_{u} \otimes \theta_{v} \otimes \theta_{\kappa}^{\vee}\right)\right) \cdot I_{d_{r}}\left(\theta_{\kappa}, \theta_{w}^{\vee}\right) .
$$

Let $\Delta: X \rightarrow X^{2}$ be the diagonal embedding. The projection formula implies that $\chi_{X^{2}}\left(\Delta_{*}\left[\theta_{X}\right] \cdot \theta_{\sigma} \otimes \theta_{\tau}^{\vee}\right)=\chi_{X}\left(\theta_{\sigma} \cdot \theta_{\tau}^{\vee}\right)=\delta_{\sigma, \tau}$ for all $\sigma, \tau \in W^{P}$, and the class 
$\Delta_{*}\left[\theta_{X}\right] \in K\left(X^{2}\right)$ is uniquely determined by this property. We deduce that (cf. [2, Thm. 3.4.1(i)])

$$
\Delta_{*}\left(\left[\theta_{X}\right]\right)=\sum_{\kappa \in W^{P}} \theta_{\kappa}^{\vee} \otimes \theta_{\kappa} \in K\left(X^{2}\right) .
$$

Since the horizontal maps are flat in the fiber square

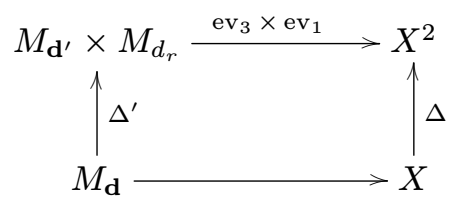

we obtain $\Delta_{*}^{\prime}\left[\theta_{M_{\mathrm{d}}}\right]=\left(\operatorname{ev}_{3} \times \mathrm{ev}_{1}\right)^{*} \Delta_{*}\left[\theta_{X}\right]=\sum_{\kappa} \operatorname{ev}_{3}^{*}\left(\theta_{\kappa}^{\vee}\right) \otimes \mathrm{ev}_{1}^{*}\left(\theta_{\kappa}\right)$. Equation (1) follows from this by another application of the projection formula.

We need the Gysin formula from [3, Thm. 3.1] stated as Proposition 5.2 below. Notice that the statement in [3] requires that the general fibers of $f$ are rational. However, this was used only to conclude that the structure sheaf of any general smooth fiber has vanishing higher cohomology, and rational connectivity suffices for this, see e.g., [10, Cor. 4.18(a)].

Proposition 5.2 ([3]). - Let $f: X \rightarrow Y$ be a surjective morphism of projective varieties with rational singularities. If the general fibers of $f$ are rationally connected, then $f_{*}\left[\theta_{X}\right]=\left[\theta_{Y}\right] \in K(Y)$.

Proof of Theorem 1. - For each sequence $\mathbf{d}=\left(d_{0}, \ldots, d_{r}\right)$ with $d_{i}>0$ for $i>0$ we choose a resolution of singularities $\pi: \widetilde{Z}_{\mathbf{d}} \rightarrow Z_{\mathbf{d}}$. By Corollary 4.7 we may assume that this resolution depends only on $|\mathbf{d}|$ and $\min \left(d_{0}, d_{X}(2)\right)$. Then form the following commutative diagram, where $M_{\mathbf{d}}^{\prime} \subset \widetilde{Z}_{\mathbf{d}} \times_{Z_{\mathbf{d}}} M_{\mathbf{d}}$ is the irreducible component mapping birationally to $M_{\mathbf{d}}$, and $\widetilde{M}_{\mathbf{d}}$ is a resolution of singularities of this component.

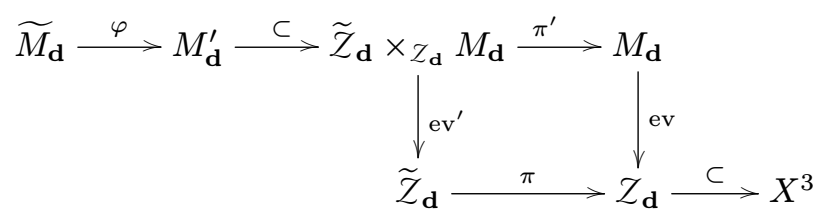

It follows from Zariski's main theorem that the fibers of $\pi^{\prime} \varphi$ are connected. Using Theorem 4.9 we deduce that the general fibers $\operatorname{of}^{\prime} \operatorname{ev}^{\prime} \varphi$ are connected. Since the map $\operatorname{ev}^{\prime} \varphi$ is smooth over a dense open subset of $\widetilde{Z}_{\mathbf{d}}$ by [15, III.10.7], it follows that the general fibers $\operatorname{of}^{\prime} \operatorname{ev}^{\prime} \varphi$ are in fact irreducible. Theorem 4.9 therefore shows that the general fibers $\operatorname{of}^{\prime} \operatorname{ev}^{\prime} \varphi$ are rationally connected, so we obtain $\left(\operatorname{ev}^{\prime} \varphi\right)_{*}\left[\theta_{\widetilde{M}_{\mathbf{d}}}\right]=\left[\theta_{\widetilde{Z}_{\mathbf{d}}}\right]$ by Proposition 5.2. Since $M_{\mathbf{d}}$ has rational singularities, we also have $\left(\pi^{\prime} \varphi\right)_{*}\left[\theta_{\widetilde{M}_{\mathbf{d}}}\right]=\left[\theta_{M_{\mathbf{d}}}\right]$. We deduce from the projection formula that

$$
\chi_{M_{\mathbf{d}}}\left(\operatorname{ev}^{*}\left(\theta_{u} \otimes \theta_{v} \otimes \theta_{w}^{\vee}\right)\right)=\chi_{\widetilde{z}_{\mathbf{d}}}\left(\pi^{*}\left(\theta_{u} \otimes \theta_{v} \otimes \theta_{w}^{\vee}\right)\right)
$$


Now Lemma 5.1 implies that

$$
\begin{aligned}
N_{u, v}^{w, d} & =\sum_{\mathbf{d}}(-1)^{r} \chi_{M_{\mathbf{d}}}\left(\mathrm{ev}^{*}\left(\theta_{u} \otimes \theta_{v} \otimes \theta_{w}^{\vee}\right)\right) \\
& =\sum_{\mathbf{d}}(-1)^{r} \chi_{\widetilde{z}_{\mathbf{d}}}\left(\pi^{*}\left(\theta_{u} \otimes \theta_{v} \otimes \theta_{w}^{\vee}\right)\right)
\end{aligned}
$$

where both sums are over all sequences $\mathbf{d}=\left(d_{0}, \ldots, d_{r}\right)$ with $d_{i}>0$ for $i>0$ and $|\mathbf{d}|=d$, and the sign $(-1)^{r}$ depends on the length of $\mathbf{d}$. Notice that the terms of the second sum depend only on $\min \left(d_{0}, d_{X}(2)\right)$ and $r$. In particular, the contributions of the sequences $\mathbf{d}=(d)$ and $\mathbf{d}=(d-1,1)$ cancel each other out. Now let $0 \leq d^{\prime} \leq d-2$. For each $r$ with $1 \leq r \leq d-d^{\prime}$, there are exactly $\left(\begin{array}{c}d-d^{\prime}-1 \\ r-1\end{array}\right)$ sequences $\mathbf{d}$ in the sum for which $d_{0}=d^{\prime}$ and the length of $\mathbf{d}$ is $r+1$. Since $\sum_{r=1}^{d-d^{\prime}}(-1)^{r}\left(\begin{array}{c}d-d^{\prime}-1 \\ r-1\end{array}\right)=0$, it follows that the corresponding terms cancel each other out. This completes the proof.

REMARK 5.3. - Theorem 1 is true also for the structure constants of the equivariant quantum $K$-theory ring $\mathrm{QK}_{T}(X)$, with the same proof. In fact, if $\widetilde{Z}_{\mathbf{d}}$ and $\widetilde{M}_{\mathbf{d}}$ are chosen to be $T$-equivariant resolutions [21, Thm. 7.6.1], then all maps used in the proofs of Lemma 5.1 and Theorem 1 are equivariant, and the arguments go through without change. More details about the ring $\mathrm{QK}_{T}(X)$ can be found in [3]. 


\section{REFERENCES}

[1] M. Brion, Positivity in the Grothendieck group of complex flag varieties, J. Algebra 258 (2002), 137-159.

[2] M. BRION, Lectures on the geometry of flag varieties, in Topics in cohomological studies of algebraic varieties, Trends Math., Birkhäuser, 2005, 33-85.

[3] A. S. Buch, L. C. Minalcea, Quantum $K$-theory of Grassmannians, Duke Math. J. 156 (2011), 501-538.

[4] A. S. Buch, Quantum cohomology of Grassmannians, Compositio Math. 137 (2003), 227-235.

[5] A. S. Buch, A. Kresch, H. Tamvakis, Gromov-Witten invariants on Grassmannians, J. Amer. Math. Soc. 16 (2003), 901-915.

[6] A. S. Buch, V. Ravikumar, Pieri rules for the $K$-theory of cominuscule Grassmannians, J. reine angew. Math. 668 (2012), 109-132.

[7] P.-E. Chaput, L. Manivel, N. Perrin, Quantum cohomology of minuscule homogeneous spaces, Transform. Groups 13 (2008), 47-89.

[8] P.-E. Chaput, N. Perrin, On the quantum cohomology of adjoint varieties, Proc. Lond. Math. Soc. 103 (2011), 294-330.

[9] P.-E. Chaput, N. Perrin, Rationality of some Gromov-Witten varieties and application to quantum K-theory, Commun. Contemp. Math. 13 (2011), 67-90.

[10] O. Debar Re, Higher-dimensional algebraic geometry, Universitext, Springer, 2001.

[11] W. Fulton, R. Pandharipande, Notes on stable maps and quantum cohomology, in Algebraic geometry - Santa Cruz 1995, Proc. Sympos. Pure Math. 62, Amer. Math. Soc., 1997, 45-96.

[12] W. Fulton, C. Woodward, On the quantum product of Schubert classes, J. Algebraic Geom. 13 (2004), 641-661.

[13] A. Givental, On the WDVV equation in quantum $K$-theory, Michigan Math. J. 48 (2000), 295-304.

[14] T. Graber, J. Harris, J. Starr, Families of rationally connected varieties, J. Amer. Math. Soc. 16 (2003), 57-67.

[15] R. Hartshorne, Algebraic geometry, Grad. Texts Math. 52, Springer, 1977.

[16] J. De Jong, J. Starr, X. He, Families of rationally simply connected varieties over surfaces and torsors for semisimple groups, preprint arXiv:0809.5224.

[17] B. Kim, R. Pandharipande, The connectedness of the moduli space of maps to homogeneous spaces, in Symplectic geometry and mirror symmetry (Seoul, 2000), World Sci. Publ., River Edge, NJ, 2001, 187-201.

[18] S. L. Kleiman, The transversality of a general translate, Compositio Math. 28 (1974), 287-297.

[19] V. L. Popov, Generically multiple transitive algebraic group actions, in Algebraic groups and homogeneous spaces, Tata Inst. Fund. Res. Stud. Math., Tata Inst. Fund. Res., 2007, 481-523.

[20] T. A. Springer, Linear algebraic groups, second ed., Progress in Math. 9, Birkhäuser, 1998. 
[21] O. E. Villamayor U., Patching local uniformizations, Ann. Sci. École Norm. Sup. 25 (1992), 629-677.

[22] F. L. ZAK, Tangents and secants of algebraic varieties, Translations of Mathematical Monographs 127, Amer. Math. Soc., 1993.

(Manuscrit reçu le 18 avril 2012;

accepté, après révision, le 5 octobre 2012.)

\author{
Anders S. BuCH \\ Department of Mathematics \\ Rutgers University \\ 110 Frelinghuysen Road \\ Piscataway, NJ 08854, USA \\ E-mail: asbuch@math.rutgers.edu \\ Pierre-Emmanuel Chaput \\ Domaine scientifique Victor Grignard \\ 239, boulevard des Aiguillettes \\ Université Henri Poincaré Nancy 1 \\ B.P. 70239 \\ F-54506 Vandœuvre-lès-Nancy Cedex, France \\ E-mail: chaput@iecn.u-nancy.fr \\ Leonardo C. MihalceA \\ Department of Mathematics \\ Virginia Tech University \\ 460 McBryde \\ Blacksburg, VA 24060, USA \\ E-mail: lmihalce@math.vt.edu \\ Nicolas Perrin \\ Mathematisches Institut \\ Heinrich-Heine-Universität \\ D-40204 Düsseldorf, Germany \\ E-mail: perrin@math .uni-duesseldorf .de
}

